\title{
Intraventricular mitral annuloplasty technique for use with repair of posterior left ventricular aneurysm
}

\author{
Igor Konstantinov, MD, ${ }^{a}$ Lynda L. Mickleborough, MD, ${ }^{a}$ James Graba, BSc, ${ }^{b}$ and Naeem Merchant, MD, ${ }^{c}$ \\ Toronto, Ontario, Canada
}

$\mathrm{P}$ osterior left ventricular aneurysms are less common than anterior aneurysms and are often associated with moderate to severe mitral insufficiency. ${ }^{1}$ Although aneurysm repair and surgical remodeling of the ventricular cavity may improve mitral valve function, mitral repair or replacement may be required in cases of severe insufficiency. We describe a new technique of intraventricular posterior patch mitral annuloplasty.

\section{Clinical Summary}

A 61-year-old woman had an inferior myocardial infarction in 1998 complicated by "pneumonia." Over the past 2 years she had increasing symptoms of fatigue, angina, and shortness of breath on exertion and occasionally at rest. She denied paroxysmal nocturnal dyspnea or orthopnea but sometimes became acutely short of breath when bending over. She had risk factors for coronary artery disease including previous smoking, hypercholesterolemia, and a positive family history. She also had peripheral vascular disease with an occluded left iliac and left subclavian artery on Doppler assessment. A preoperative echocardiogram showed a grade III ventricle with a large basal inferior aneurysm and moderate to severe mitral regurgitation (Figure 1, A). Cardiac catheterization showed a posterior aneurysm measuring $6 \times 8 \mathrm{~cm}^{2}$ and triple vessel disease. She had a $50 \%$ stenosis of the left anterior descending coronary artery with a $70 \%$ stenosis of the right coronary artery. The distal circumflex vessel was totally occluded with no vessel seen beyond the occlusion. A preoperative magnetic resonance imaging scan showed an end-diastolic volume of $243 \mathrm{~mL}$ with an ejection fraction of $26 \%$ (Figure 2, A). She was considered a candidate for revascularization, repair of the posterior aneurysm, and mitral annuloplasty.

At surgery, a transesophageal echocardiogram revealed normal appearing mitral leaflets but a dilated anulus measuring $39 \mathrm{~mm}$

From the Divisions of Cardiac Surgery, ${ }^{\mathrm{a}}$ Cardiology, ${ }^{\mathrm{b}}$ and Radiology, University Health Network, University of Toronto, Toronto, Ontario, Canada.

Received for publication March 15, 2001; accepted for publication April 17, 2001.

Address for reprints: L. L. Mickleborough, MD, Toronto General Hospital, EN 13-217, 200 Elizabeth St, Toronto, Ontario M3A 2S1, Canada (E-mail: lynda.mickleborough@uhn.on.ca).

J Thorac Cardiovasc Surg 2001;122:1244-7

Copyright () 2001 by The American Association for Thoracic Surgery $0022-5223 / 2001 \$ 35.00+0 \quad \mathbf{1 2 / 5 4 / 1 1 6 5 4 8}$

doi: $10.1067 / \mathrm{mtc} .2001 .116548$
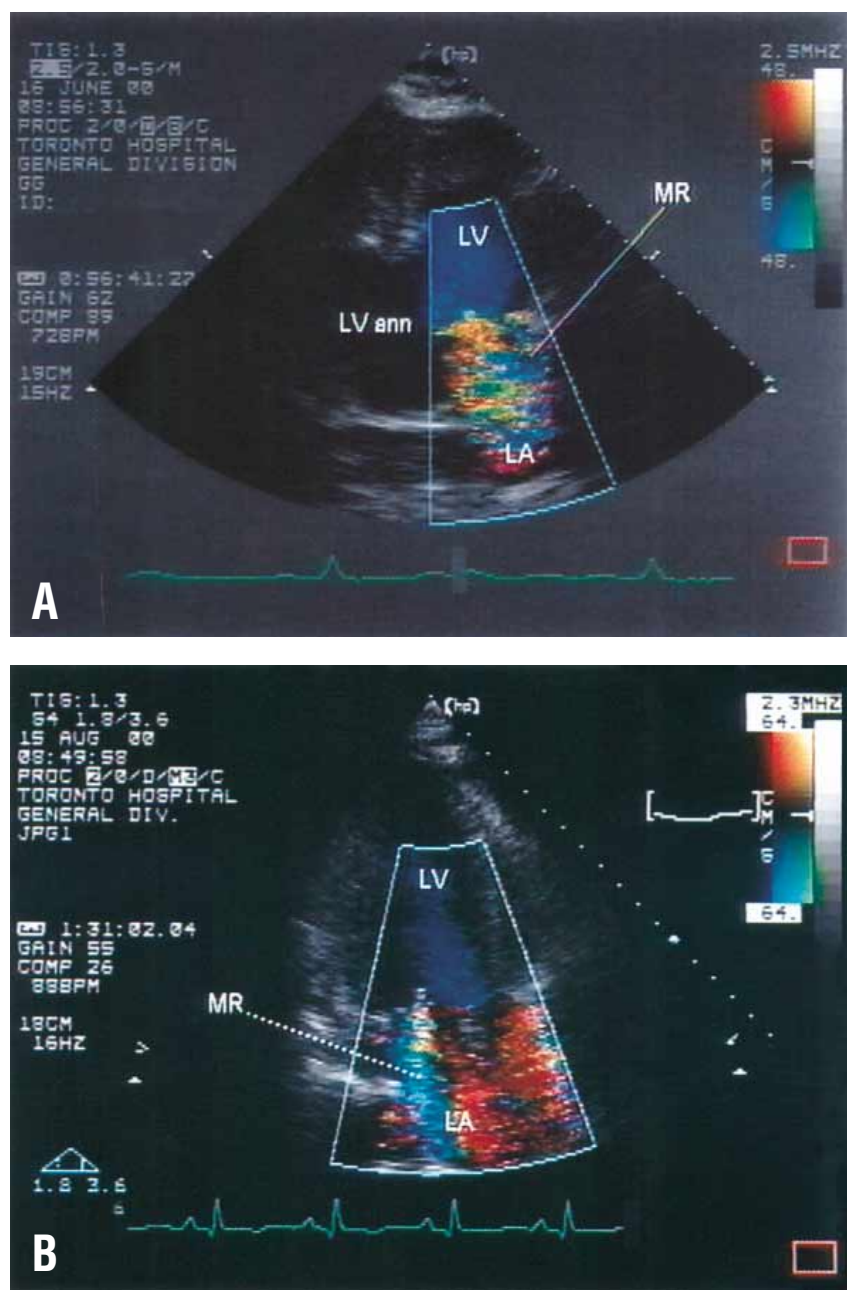

Figure 1. A, Preoperative transthoracic echocardiogram, apical long-axis view, showing moderate to severe mitral regurgitation. B, Similar view postoperatively showing mild mitral regurgitation. $L V$, Left ventricle; $L A$, left atrium; $M R$, mitral regurgitation.

(Figure $3, A$ ). The heart was elevated through a midline sternotomy during standard cardiopulmonary bypass (Figure $4, A$ ). The area of prior infarct expansion and thinning was just lateral to the posterior interventricular artery and medial to the area of insertion of the posterior papillary muscle (Figure $4, B$ ). The posterior aneurysm was opened and layered thrombus was carefully removed. Most of the thinned left ventricular wall was resected except for a small 

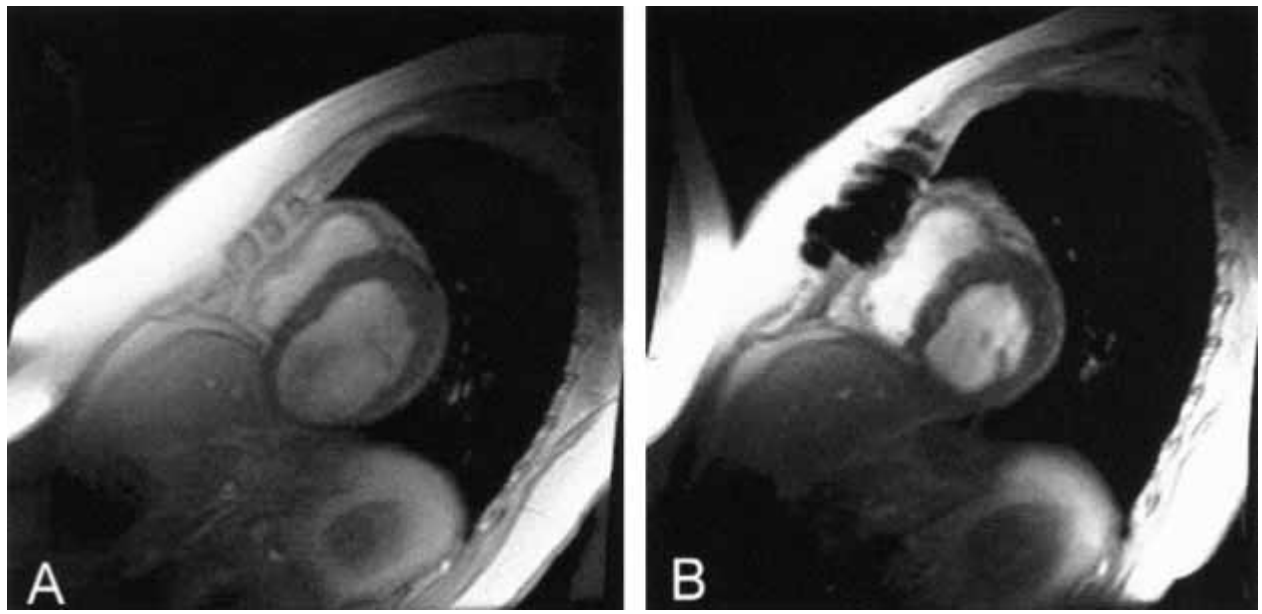

Figure 2. A and B, Preoperative and postoperative magnetic resonance imaging scans illustrating decreased ventricular size resulting from successful mitral annuloplasty and posterior aneurysm repair.
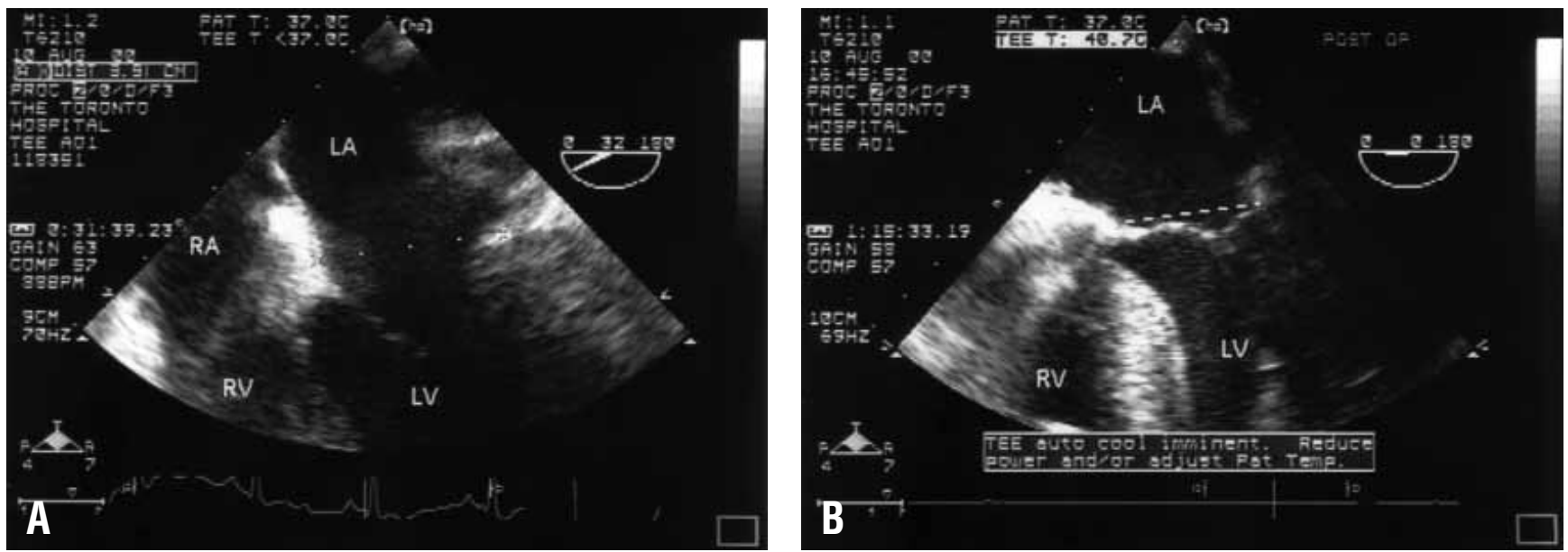

Figure 3. A and B, Transesophageal intraoperative echocardiogram, 4-chamber views, to assess anulus diameter before and after repair ( $39 \mathrm{~mm}$ vs $30 \mathrm{~mm}$ ). $R V$, Right ventricle; $R A$, right atrium; $L V$, left ventricle; $L A$, left atrium.

area just under the posteromedial portion of the mitral anulus (Figure 4, C). To reinforce this area and to perform the intraventricular annuloplasty, we used a patch of preserved bovine pericardium. The size of the patch was determined by the size of the thinned posterior wall defect and by the amount of plication of the posterior anulus that we wanted to achieve. The degree of plication was determined by visual assessment and was not amenable to a specific formula or calculation. In this case, approximately half of the posterior mitral anulus was involved in the area of thinning, and the edge of the patch required to achieve the desired plication of the anulus measured $25 \mathrm{~mm}$.

The medial portion of the mitral anulus was plicated as the patch was attached (Figure 4, $D$ and $E$ ). The suture line was then carried onto the preserved portion of the posterior septum and posterior free wall so as to exclude and reinforce the thinned portion of the posterior wall and septum, as previously described (Figure $4, F) .^{2}$ The distal end of the patch was incorporated into the linear closure of the aneurysm (Figure 4, $F$ and $G$ ). Deairing was carried out through a vent in the right superior pulmonary vein and through the incision as the closure was completed.

A crossclamp was then applied and cardioplegic arrest was achieved. Saphenous vein grafts were placed to the left anterior descending and right coronary arteries. The patient was easily weaned from bypass. Transesophageal echocardiography in a loaded state (systolic blood pressure $150 \mathrm{~mm} \mathrm{Hg}$ ) showed that the mitral anulus diameter measured $30 \mathrm{~mm}$ (Figure 3,B), and mitral insufficiency was mild. Her postoperative course was uneventful and she was discharged to her home on the seventh postoperative day. Follow-up magnetic resonance imaging 2 months after the operation showed a grade II ventricle with an ejection fraction of $55 \%$ and an end-diastolic volume of $118 \mathrm{~mL}$ (Figure 2, B). A postoperative echocardiogram showed only mild mitral regurgitation (Figure 1, B). Further follow-up at 6 months confirmed durability of the repair. 

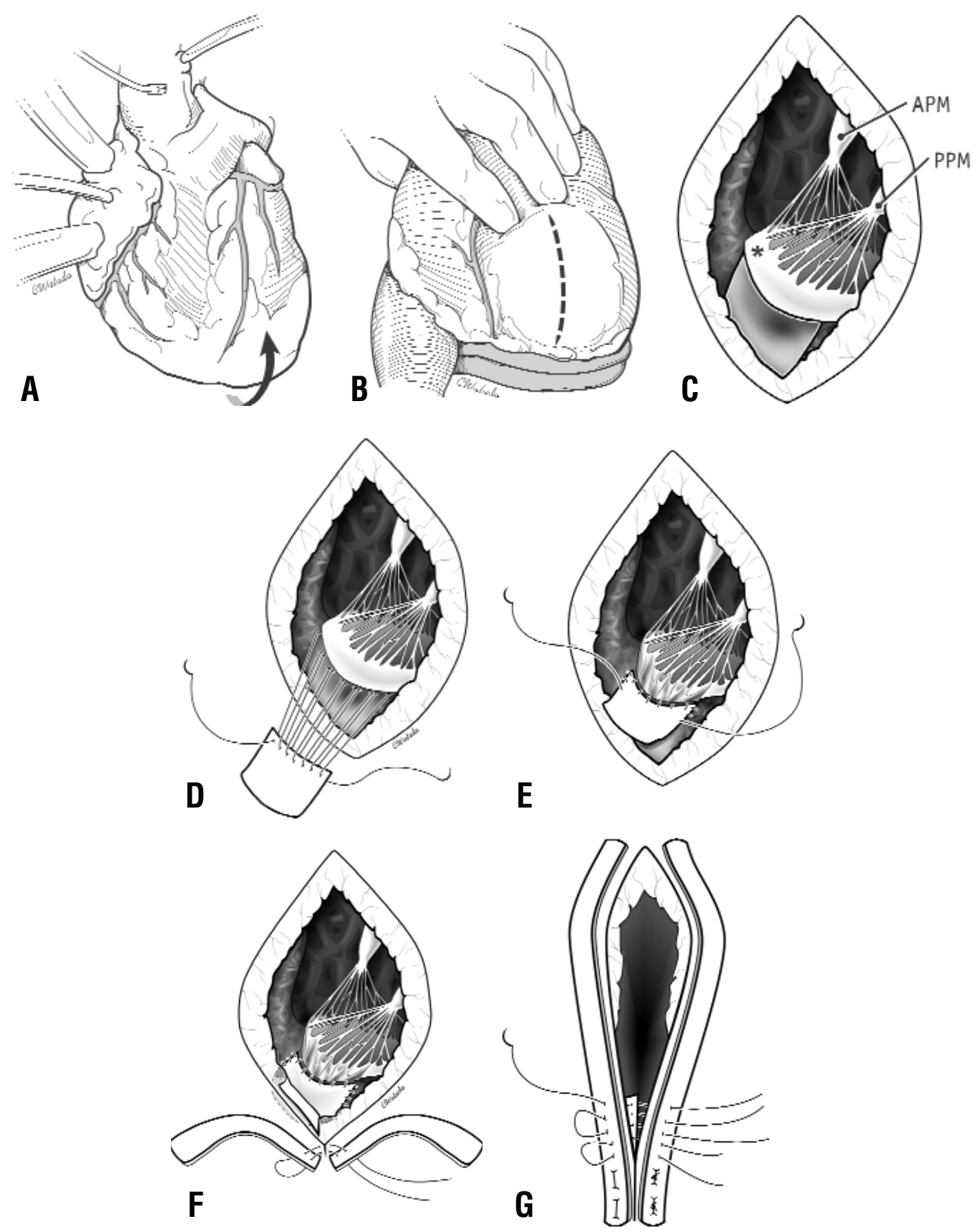

Figure 4. Diagram of operative technique. A, During cardiopulmonary bypass the apex of the heart is elevated. B, The area of infarct expansion and thinning is shown just lateral to the posterior interventricular artery and medial to the area of insertion of the posterior papillary muscle. The dashed line indicates the proposed ventriculotomy incision. C, The thinned portion of the wall has been excised except for the portion directly adjacent to the posteromedial mitral anulus, as indicated by the area outlined in the diagram. Only a portion of the mitral valve anulus can be seen through the incision. The anterior papillary muscle (APM) and posterior papillary muscle (PPM) are indicated. The asterisk indicates the region of the posteromedial commissure of the mitral valve. D, Sutures are inserted to plicate a portion of the posterior mitral anulus with a pericardial patch. $E$, The patch has been lowered into position. Note plication of a portion of the valve leaflet as indicated in the diagram. The suture line is then continued below the anulus onto the preserved part of the posterior septum and posterior free wall so as to exclude and reinforce the thinned portion of the posterior wall outlined in panel $C$. $F$, The distal end of the patch is brought out through the defect in the posterior wall. G, The distal edge of the patch is incorporated into the posterior linear closure, which is reinforced with felt strips. 


\section{Comment}

The influence of aneurysm repair on concomitant mitral regurgitation has been previously discussed. ${ }^{1,2}$ In our series of 29 posterior aneurysms, 7 patients or $24 \%$ had severe ( 3 or $4+$ ) mitral regurgitation before the operation. In 5 patients, a posterior aneurysm repair was carried out but no attempt was made to plicate the mitral anulus during the repair. In all 5, the mitral regurgitation improved by at least 1 grade after the operation. In these cases, improved mitral valve function may have been due to (1) decreased dilatation of the anulus secondary to decreased left ventricular size, (2) improved function of ischemic papillary muscles with revascularization, or (3) realignment of the papillary muscles associated with posterior ventricular repair.

One patient with 4+ mitral regurgitation underwent valve replacement in addition to posterior aneurysm repair, and the final patient is the one included in this report, who underwent a mitral valvuloplasty. Further long-term follow-up of these patients is needed to determine which surgical approach provides optimal results in patients with mitral regurgitation and a posterior aneurysm.

$\mathrm{We}^{2}$ have previously described a technique for repair of posterior aneurysms using an endoventricular pericardial patch. The current report describes how such a patch can be used to achieve an intraventricular posterior mitral valvuloplasty at the same time as aneurysm repair. Advantages of this approach are that repair of the aneurysm and valvuloplasty can be accomplished through a single incision. By using a single patch in the open beating heart, we can minimize ischemic time and pump time, which may be important in patients with poor left ventricular function and diffuse coronary artery disease.

Thanks to S. Siu, MD, for assistance in echocardiographic assessment of the patient, to Cynthia Yee for her excellent illustrations, and to Hilary Vincent for preparation of the manuscript.

\section{References}

1. Mickleborough LL, Carson S, Ivanov J. Repair of dyskinetic or akinetic left ventricular aneurysm: results obtained with a modified linear closure. J Thorac Cardiovasc Surg. 2001;121:675-82.

2. Mickleborough LL. Surgical management of left ventricular aneurysms. Sem Thorac Cardiovasc Surg. 1995;7:233-9. 